\title{
THERMAL EFFECTS ON CAMERA FOCAL LENGTH IN MESSENGER STAR CALIBRATION AND ORBITAL IMAGING
}

\author{
S. Burmeister ${ }^{1}$, S. Elgner ${ }^{2}$, F. Preusker ${ }^{2}$, A. Stark ${ }^{2}$, J. Oberst ${ }^{1.2}$ \\ ${ }^{1}$ Technical University Berlin, Institute for Geodesy and Geoinformation Sciences, Berlin, Germany - \\ (steffi.burmeister, juergen.oberst)@tu-berlin.de \\ ${ }^{2}$ German Aerospace Center (DLR), Institute of Planet. Research, Berlin, Germany - \\ (stephan.elgner, frank.preusker, alexander.stark, juergen.oberst)@dlr.de
}

Commission VI, WG VI/4

KEY WORDS: Mercury, MESSENGER, MDIS Camera Digital Terrain Models

\begin{abstract}
:
We analyse images taken by the MErcury Surface, Space ENviorment, GEochemistry, and Ranging (MESSENGER) spacecraft for the camera's thermal response in the harsh thermal environment near Mercury. Specifically, we study thermally induced variations in focal length of the Mercury Dual Imaging System (MDIS). Within the several hundreds of images of star fields, the Wide Angle Camera (WAC) typically captures up to 250 stars in one frame of the panchromatic channel. We measure star positions and relate these to the known star coordinates taken from the Tycho-2 catalogue. We solve for camera pointing, the focal length parameter and two non-symmetrical distortion parameters for each image. Using data from the temperature sensors on the camera focal plane we model a linear focal length function in the form of $f(T)=A_{0}+A_{1} T$. Next, we use images from MESSENGER's orbital mapping mission. We deal with large image blocks, typically used for the production of a high-resolution digital terrain models (DTM). We analyzed images from the combined quadrangles $\mathrm{H} 03$ and H07, a selected region, covered by approx. 10,600 images, in which we identified about 83,900 tiepoints. Using bundle block adjustments, we solved for the unknown coordinates of the control points, the pointing of the camera - as well as the camera's focal length. We then fit the above linear function with respect to the focal plane temperature. As a result, we find a complex response of the camera to thermal conditions of the spacecraft. To first order, we see a linear increase by approx. $0.0107 \mathrm{~mm}$ per degree temperature for the Narrow-Angle Camera (NAC). This is in agreement with the observed thermal response seen in images of the panchromatic channel of the WAC. Unfortunately, further comparisons of results from the two methods, both of which use different portions of the available image data, are limited. If leaving uncorrected, these effects may pose significant difficulties in the photogrammetric analysis, specifically these may be responsible for erroneous longwavelength trends in topographic models.
\end{abstract}

\section{INTRODUCTION}

The MErcury Surface, Space ENvironment, GEochemistry, and Ranging (MESSENGER) spacecraft entered orbit about Mercury in March 2011 to carry out a comprehensive mapping of the planet in its harsh thermal environment, close to the Sun. Due to MESSENGER's highly elliptical orbit solar irradiation and thermal flux from the planet vary greatly during one orbital pass. The spacecraft is equipped with the Mercury Dual Imaging System (MDIS), including a Wide Angle Camera (WAC) and a Narrow-Angle Camera (NAC) which both appear to be very much affected by changing thermal conditions (Denevi et al., 2017; Hawkins et al., 2007). We use two distinct approaches to study and derive functional descriptions of the thermal impact and focal length behaviour of the camera.

\section{STAR-FIELD IMAGING}

During the cruise phase, the spacecraft took several hundreds of images of star fields. The WAC's panchromatic channel may capture up to 250 stars in one frame, depending on chosen exposure time. As exposure times (chosen to minimize noise levels and dark current effects) are dictated by temperature and radiation, only few stars are seen in the images taken at high temperature, thus leading to larger errors in estimates of camera parameters. Also, only few stars are seen in images of the (narrow-band) color channels, which were excluded from the analysis. Also, the number of visible stars in the Narrow-Angle
Camera (NAC) images is not sufficient for estimates of parameters of the NAC. The MDIS camera system is equipped with several temperature sensors, and temperature of e.g. the focal plane is readily available from the image housekeeping data. Temperatures of the focal plane range from approx. $-40^{\circ} \mathrm{C}$ during the cruise phase to approx. $+20^{\circ} \mathrm{C}$ when the spacecraft moves close to Mercury.

We measure star positions in the images with subpixel-accuracy and relate these to the known star coordinates taken from the Tycho-2 catalogue by using the nominal camera pointing and intrinsic parameters. In total, 260 images were used in separate least-squares-fits to solve for individual camera pointing parameters, and one camera focal length parameter. We also solve for two parameters describing non-symmetrical distortions. We assume a standard pin-hole camera model complemented by terms describing non-symmetrical distortions:

$$
\begin{aligned}
& d x=2 B_{2} x y+B_{1}\left(r^{2}+2 x^{2}\right) \\
& d y=2 B_{1} x y+B_{2}\left(r^{2}+2 y^{2}\right),
\end{aligned}
$$

where $\quad d x, d y=$ image coordinate correction

$x, y=$ image coordinates

$x_{0}, y_{0}=$ image coordinates of the principal point

$B_{1}, B_{2}=$ non-symmetrical distortion parameters

and

$$
r=\sqrt{\left(x-x_{0}\right)^{2}+\left(y-y_{0}\right)^{2}},(\text { Brown, 1971). }
$$

* Corresponding author 
The principal point coincides with the image centre and the average values of all results for $B_{1}$ and $B_{2}$ after the fit are:

$$
\begin{aligned}
& B_{1}=-2.84 \times 10^{-5} \pm 5.92 \times 10^{-6} \\
& B_{2}=-2.11 \times 10^{-7} \pm 5.90 \times 10^{-6} .
\end{aligned}
$$

Geometric distortions amount to up to 0.47 pixels in the image corners (Fig. 1) with $B_{1}$ having the considerably larger impact on the image coordinates. With its large error after the fit, parameter $B_{2}$ is found to be not significant in our model.

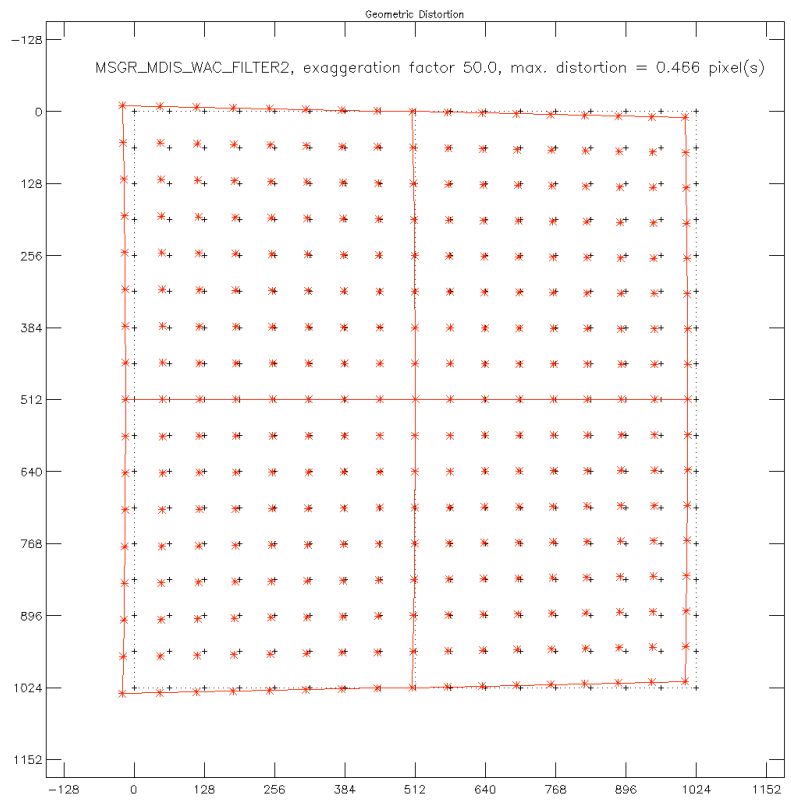

Figure 1: Geometric distortion of WAC Filter B (exaggerated by a factor of 50 ).

We study the effect of temperature to find that an increase in temperature leads to an increasing focal length value (Fig. 2). However, it appears that the geometric distortion (i.e., described by the parameter $B_{1}$ above) is not affected by temperature. The average RMS of all results combined after the fit is $0.11 \pm 0.03$ pixels.

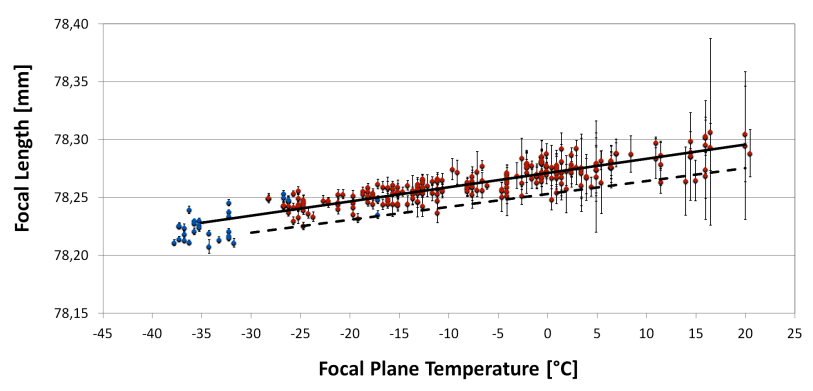

Figure 2: Focal length in relation to focal plane temperature. Error bars depict focal length accuracy after the fit. Results from images taken during the cruise are shown in blue; results from orbit are shown in red. Solid black line shows our linear function, while the dashed line is based on results given in (Denevi et al., 2017).

To give a functional description between temperature $T$ and focal length $f$, we introduce a linear expression in the form of $f(T)=A_{0}+A_{1} T$, where $T$ is measured in ${ }^{\circ} \mathrm{C}$ and $f$ is measured in $\mathrm{mm}$. In practice, $A_{0}$ depicts the focal length value at $0^{\circ} \mathrm{C}$ focal plane temperature. We can solve for $A_{0}$ and $A_{1}$ in a leastsquares-fit by using the individual focal length results and the corresponding CCD plane temperatures and find:

$$
\begin{aligned}
& A_{0}=78.2712 \pm 0.0035 \mathrm{~mm} \\
& A_{1}=1.23 \times 10^{-3} \pm 2.04 \times 10^{-4} \mathrm{~mm} /{ }^{\circ} \mathrm{C}
\end{aligned}
$$

We think that this linear function is sufficiently practical to describe the change in focal length within the observed temperature range.

When comparing our results with those given in Denevi et al. (2017), we find that the slopes of both linear functions are similar, but that there is a constant offset of approx. $0.02 \mathrm{~mm}$ (Fig 2). This discrepancy is probably explained with the use of a different geometric distortion model affecting the focal length value.

\section{BUNDLE BLOCK ADJUSTMENTS}

Next, we take an alternative approach and use surface images from MESSENGER's orbital mission. We are concerned with the production of a high-resolution digital terrain models (DTM) from large image blocks (see companion abstract at this conference by Preusker et al. 2018). The MDIS images provide at least triple coverage for almost all areas on Mercury at a resolution better than $350 \mathrm{~m} / \mathrm{pixel}$. We analysed images within the connected quadrangles $\mathrm{H} 03$ and $\mathrm{H} 07$ (cf. Preusker et al., 2018), covered by approx. 10,600 images, among which we identified about 50,000 stereo image combinations, consisting of at least three images each. Here, we measured approx. 83,900 tie-points.

\subsection{Functional approach}

We carried out bundle-block adjustments and solved for the unknown coordinates of the control points, the pointing of the camera, as well as the camera's focal length. The focal length is introduced as an unknown parameter for each image according to a specific adjustment technique, where we solve for so-called "Observed Unknowns". The camera pointing uncertainties (which are critical for the adjustment results) are 0.5 degree for NAC and 0.15 degree for WAC. The spacecraft orbit is assumed fixed, to avoid interference with focal length effects. (Specifically, spacecraft range is highly correlated with focal length).

\subsection{Results}

Similar as in the star calibrations, we introduce a simple linear function to describe the focal length of NAC and WAC and dependence on temperature:

$$
f(T)=A_{0}+A_{1} T,
$$

where $\quad T=$ temperature measured in ${ }^{\circ} \mathrm{C}$

$f=$ focal length measured in $\mathrm{mm}$

$A_{0}, A_{1}=$ regression coefficients.

The obtained regression coefficients are shown in Table 1. As outlined in Figure 3 the averaged focal-length values obtained for the individual images are following a clear trend with increasing temperature. The strong scatter of the values for the individual images might be due to residual mis-modelling of the 

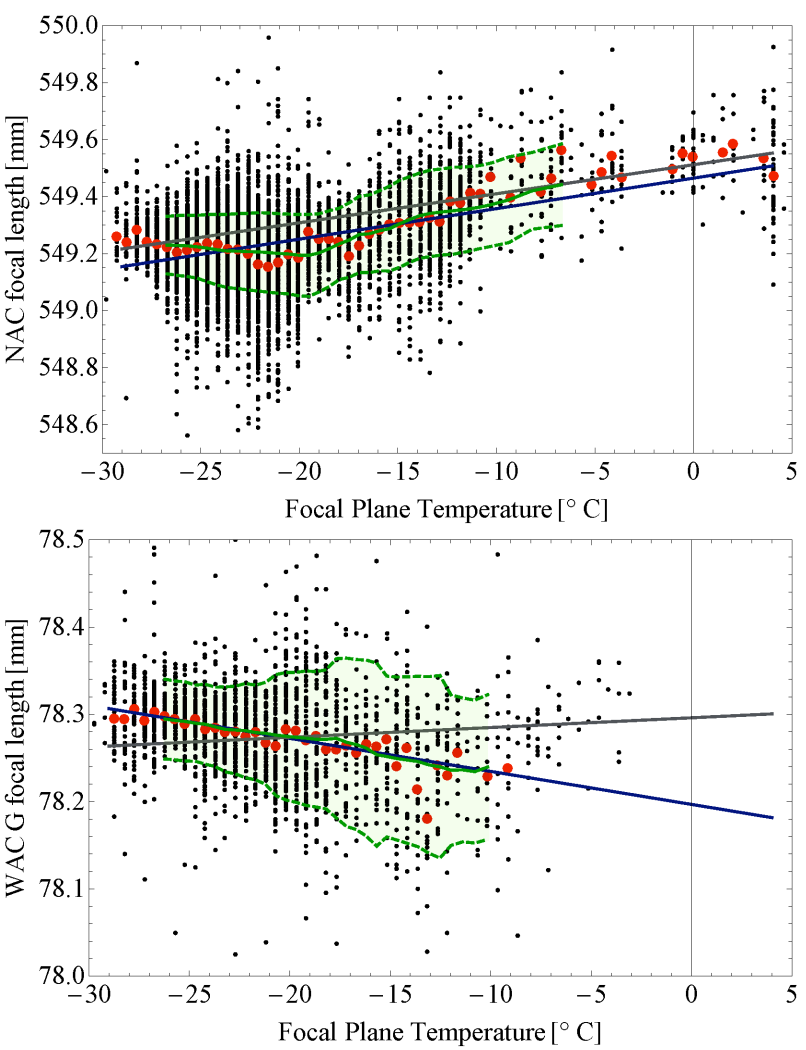

Fig. 3: NAC and WAC (Filter G) focal length as function of focal-plane temperature. The black dots denote the focal lengths of the images obtained through bundle-block adjustment. The red dots are mean values of focal lengths of the same temperature. Note: The precision of the temperature measurement is only $0.5^{\circ} \mathrm{C}$. Mean values were not calculated for temperature regime with data from less than 5 images. The green line denotes the averages of the focal lengths within temperature bins of 5 degrees. The green shaded area with the dashed green lines depicts the standard deviation within the temperature bins. The blue line is a linear fit to the green curve, while the standard deviations of the binned data were used as weights. The grey line is the model proposed by Denevi et al. (2017). (Note: Denevi et al. (2017) list $1.117 \times 10^{-2} \mathrm{~mm} /{ }^{\circ} \mathrm{C}$ for the WAC focal length effect, probably a typo. The corresponding instrument SPICE kernel to which the authors refer shows $1.117 \times 10^{-3} \mathrm{~mm} /{ }^{\circ} \mathrm{C}$. The figure shows the value reported in the instrument kernel.)

\begin{tabular}{|l|c|c|}
\hline Camera & $A_{0}$ & $A_{1}$ \\
\hline & $\mathrm{mm}$ & $\mathrm{mm} /{ }^{\circ} \mathrm{C}$ \\
\multirow{3}{*}{ WAC (Filter G) } & $78.2012 \pm 0.0028$ & $-0.00359 \pm 0.00013$ \\
\cline { 2 - 3 } NAC & $549.465 \pm 0.020$ & $0.0107 \pm 0.0010$ \\
\hline
\end{tabular}

Tab. 1: Fit parameters for focal length function (Eq. 4)

Parameters were obtained from a least-squares fit to measurements binned into $5^{\circ}$ temperature ranges and weighted by the variance within the bin.

camera alignment and distortion but also due to spacecraft pointing and trajectory errors. The temperature dependence for the NAC is in a good agreement with the model described by Denevi et al. (2017). While the slope in their model $\left(1.019 \times 10^{-2}\right.$ $\mathrm{mm} /{ }^{\circ} \mathrm{C}$ ) differs to ours $\left(A_{1}\right)$ by only $51 \mu \mathrm{m} /{ }^{\circ} \mathrm{C}$., their curve intercept (i.e., $A_{0}$ ) is offset by $47 \mu \mathrm{m}$. This is larger than our uncertainty for $A_{0}$ but might be related to systematic effects related to the study area or the lack of observations at higher temperatures. The temperature dependence for the WAC filter $\mathrm{G}$ is puzzling. The focal length seems to decrease with temperature, while Denevi et al. (2017) and our findings from the star calibration report a slight increase of the focal length with temperature. One possible explanation could be that the panchromatic filter (B) used in the star calibration and the filter $\mathrm{G}$ used in the mapping have a different response to temperature. This might involve a different physical characteristic of the filter material and/or a modified distortion function. Further investigations involving more image data with an extended temperature range might help to shed more light into this issue.

\section{CONCLUSION}

Using the two described techniques, we find a complex response of the MESSENGER camera to thermal conditions of the spacecraft. For both techniques, we attempt to model linear effects. From the star calibration of WAC images taken by the panchromatic filter, we model a linear increase in focal length with temperature, in reasonable agreement with previous calibration efforts (Denevi et al., 2017). No calibration can be carried out for other WAC filters or the NAC, due to lack of sufficient numbers of stars. From block adjustments, we find an increase by $0.0107 \mathrm{~mm}$ per degree temperature for NAC. In contrast, for the WAC filter G we rather see a decrease of focal length with temperature. Unfortunately, as no mapping was possible with the highly sensitive panchromatic channel of the WAC, no calibration with this technique in the context of block adjustments can be carried out.

If leaving uncorrected, thermal effects on focal length may pose significant difficulties in the photogrammetric analysis. Specifically these may be responsible for erroneous longwavelength trends in topographic models. We currently investigate higher-order effects of thermal anomalies in the pointing alignment of the camera.

\section{ACKNOWLEDGEMENTS (OPTIONAL)}

S.B. and J.O. were supported by a grant from the German Science Foundation (DFG), GZ: OB 124/11-1.

\section{REFERENCES}

Brown, D.C., 1971. Close-range camera calibration. Photogramm. Eng 37, 855-866.

Denevi, B.W., Chabot, N.L., Murchie, S.L., Becker, K.J., Blewett, D.T., Domingue, D.L., Ernst, C.M., Hash, C.D., Hawkins, S.E., Keller, M.R., Laslo, N.R., Nair, H., Robinson, M.S., Seelos, F.P., Stephens, G.K., Turner, F.S., Solomon, S.C., 2017. Calibration, Projection, and Final Image Products of MESSENGER's Mercury Dual Imaging System. Space Sci Rev $214,2$.

Hawkins, I., S. Eduard, Boldt, J.D., Darlington, E.H., Espiritu, R., Gold, R.E., Gotwols, B., Grey, M.P., Hash, C.D., Hayes, J.R., Jaskulek, S.E., Kardian, C.J., Jr., Keller, M.R., Malaret, E.R., Murchie, S.L., Murphy, P.K., Peacock, K., Prockter, L.M., Reiter, R.A., Robinson, M.S., Schaefer, E.D., Shelton, R.G., Sterner, I., Raymond E., Taylor, H.W., Watters, T.R., Williams, B.D., 2007. The Mercury Dual Imaging System on the MESSENGER spacecraft. Space Sci Rev 131, 247-338.

Revised March 2018 